\title{
Deep Learning and Implementations in Banking
}

\begin{abstract}
Data-driven technologies have been changing every aspect of human life and the fast-developing banking sector with its data rich nature has become the implementation field of these fastevolving technologies. Deep learning, as one of the emerging technology in recent years, has also been inevitably adopted for various improvements in banking. To the best of our knowledge, there is no comprehensive literature review which focuses on specifically deep learning and its implementations in banking. Therefore, this paper investigates the deep learning technology in depth and summarizes the relevant applications in banking so to contribute to the existing literature. Moreover, by providing reliable and up to date review, it is also aimed to serve as the one-stop repository for banks and researchers who are interested in embracing deep learning, whilst bringing insights for the directions of future research and implementation.
\end{abstract}

Keywords: Deep Learning; Banking; Implementations; Trends; Challenges.

\section{Introduction}

It has been proven inevitable that almost all science subjects are embracing the data-driven decision making and catching up them with the rapidly evolving data-driven technologies [1-4]. Under the thick blanket of overflowing Big Data, the usually hidden and significantly valuable information are encouraging numerous researchers and practitioners with relevant skills to further improve the capability of nowadays data science empowered by advancing Artificial Intelligence (AI) technologies. As one of the most influential subjects that closely relates to almost everything of people's day-to-day life, banking has been one of the pioneering sectors that actively develop and implement advanced data-driven technologies. Thanks to the data-rich nature of 
the banking sector as well as the well developed essential data science infrastructure, banking has been a popular field for data-driven researches on a global scale $[3,5]$.

One may notice that the variety of terms like machine learning, data mining, deep learning, AI are used interchangeably, especially by the numerous resources made available online. This is the inevitable result of the growing interests of the general public, the rapid developments of these advanced technologies themselves, and the general knowledge gap due to the lack of reliable educational resources in scale. The tech-knowledge gap and its associated problems have been developing faster than ever nowadays, which has already become the challenge for scaling up of many newly emerging industries, not to mention the consequences of fraud-related criminal activities due to being lack of knowledge or most up to date information. Thus, it is crucially important to address the real meaning of these terms and summarize the most up to date progresses.

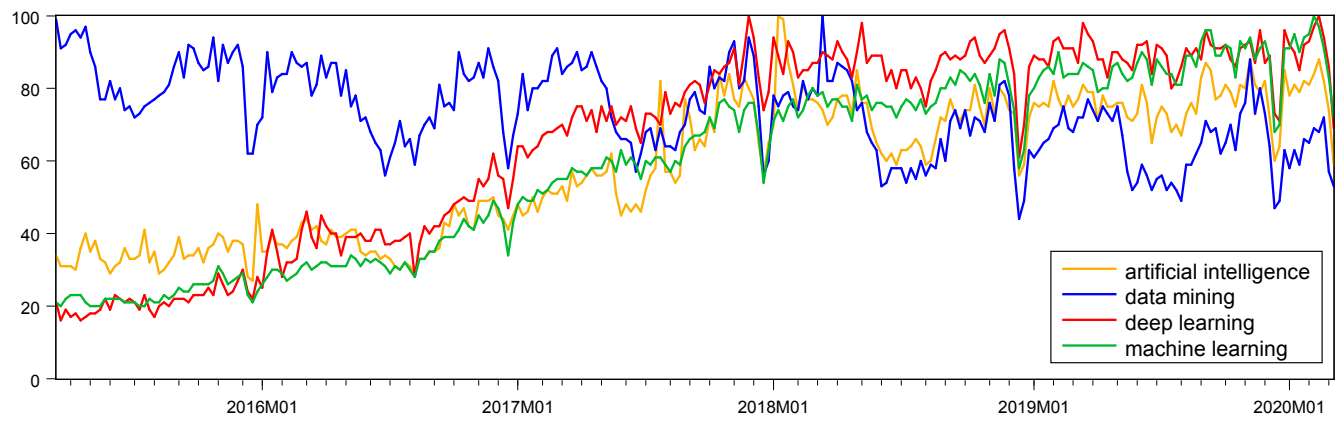

Figure 1: Global Google Trends from 2014 March to 2020 March.

As can be seen in Figure 1, where the monthly global Google trends indices are collected for the terms including AI, data mining, machine learning and deep learning since 2014. Although data mining attracted much more attention globally from the beginning in comparison with the other three and showed slightly downward declining trend, yet it is still maintaining a relatively good level of public interests. AI, machine learning and deep learning have all been rapidly growing over the years, where deep learning shows the steepest exponentially growing curve and stays the highest position closely followed by machine learning and AI. This further confirmed the shift of attention due to the rapid advancements of these closely connected technologies in general. It also further supports the main aim of this paper, which is to thoroughly investigate 
this emerging domain of deep learning and its implementations in the high profile banking sector.

Regarding the existing literature, there are a few comprehensive review papers which focus on the data mining applications perspective $[3,6-8]$, few papers further investigated some specific implementations of data mining in banking, e.g. customer relationship management (CRM) [9], fraud detection [10], credit scoring [11], etc. There are also researches which addressed the big data implementations in banking sector [12], [5] investigated the blockchain-ed big data impact in banking, whilst authors in [13] presented a specific analysis for Indian banks. Moreover, some researchers addressed machine learning techniques in credit rating [14], risk management $[15,69]$, customer profiling and segmentation $[17,18]$. However, it is to the best of our knowledge that there is no comprehensive review focusing on deep learning and its implementations in banking. Considering the emerging interests of deep learning and fast developing of the banking sector, this paper is of paramount importance to connect the researchers of both subjects, summarize the most up to date implementations and offer insights for future research directions.

The rest of the paper is organized so that section 2 introduces deep learning in-depth, section 3 summarizes the implementations of deep learning in the banking sector, finally, the conclusion is provided in section 4 .

\section{Deep Learning}

Deep learning is an emerging branch of machine learning as evident by its peaking trend index in Figure 1. There have been tremendous acknowledgments of its advancements comparing to other relatively "less deep" machine learning techniques. It is noticed that the knowledge gap is still the most significant entry barrier for most researchers and practitioners who are interested in adopting more advanced technologies. This section will introduce deep learning and its key techniques in details and it is also aimed to provide general and clear guidance of understanding the differences among terms including AI, machine learning and deep learning, which are many times used interchangeably.

Most people start to notice the rising of AI over the last decade, whilst AI is actually a long history field of research back to the 1950s. For any software, programs, platforms and machines, the capabilities of processing and learning driven by simulating the human intelligence can be generally referred to as AI. The features of AI trending nowadays are the joint efforts of rapid technological revolution and the expanding robotic process automation market [19]. 
Undoubtedly, as witnessed by the changes AI has brought to our day to day life, AI nowadays in the format of automated objects, process and augmented analytics are the keys of success across sectors and subjects.

In general, AI as a much broader umbrella term contains many subsets: expert systems, natural language processing, robotics, planning, speech and vision processing, as well as machine learning, which is a significant subset of AI (the branches of AI can be found in [19]). It is of note that the challenges remain to keep on top of all advancements as every branch involved are rapidly evolving in the dimensions of both themselves and the combinations with one or multiple other branches or trending techniques. In brief, machine learning as an important subset of AI refers to the independent learning ability endowed to technological equipment on data so to allow knowledge acquisition and performance improvements. Specifically, machine learning algorithms enable the tasks to be achieved sufficiently by learning to generalize from data or examples [20]. Considering the levels of involvement of human experiences or judgements in the training process, machine learning can be further divided into supervised learning, semisupervised learning, unsupervised learning, and reinforcement learning [21]. More details of a variety of machine learning algorithms can be found in established literature $[22,23,46]$, thus we will not repeat here.

Many times the terms deep learning and machine learning are used interchangeably and it is noticed to be relatively blurry in clearly distinguishing these two. Technically speaking, deep learning is machine learning, and it is considered to be a subset of machine learning where different flavours of neural network techniques are incorporated. Deep learning techniques have very strong intuitions of independent decision making and they are inspired by the mechanism of the neural network of the human brain. The key difference of deep learning is that the machine learns different layers of features following a logical structure from data on its own rather than accepting the design of human engineers [25]. For instance, the AlphaGo by Google has demonstrated a great example of deep learning. The idea of endowing machine the ability to solitarily learn and solve problem on its own has further promoted the development and implementation of deep learning across sectors [26-30]. Although it is still a questionable debate, many argue that the advancements of deep learning will lead to "true AI" whilst others disagree considering the supervision and labelled data required by deep learning. 


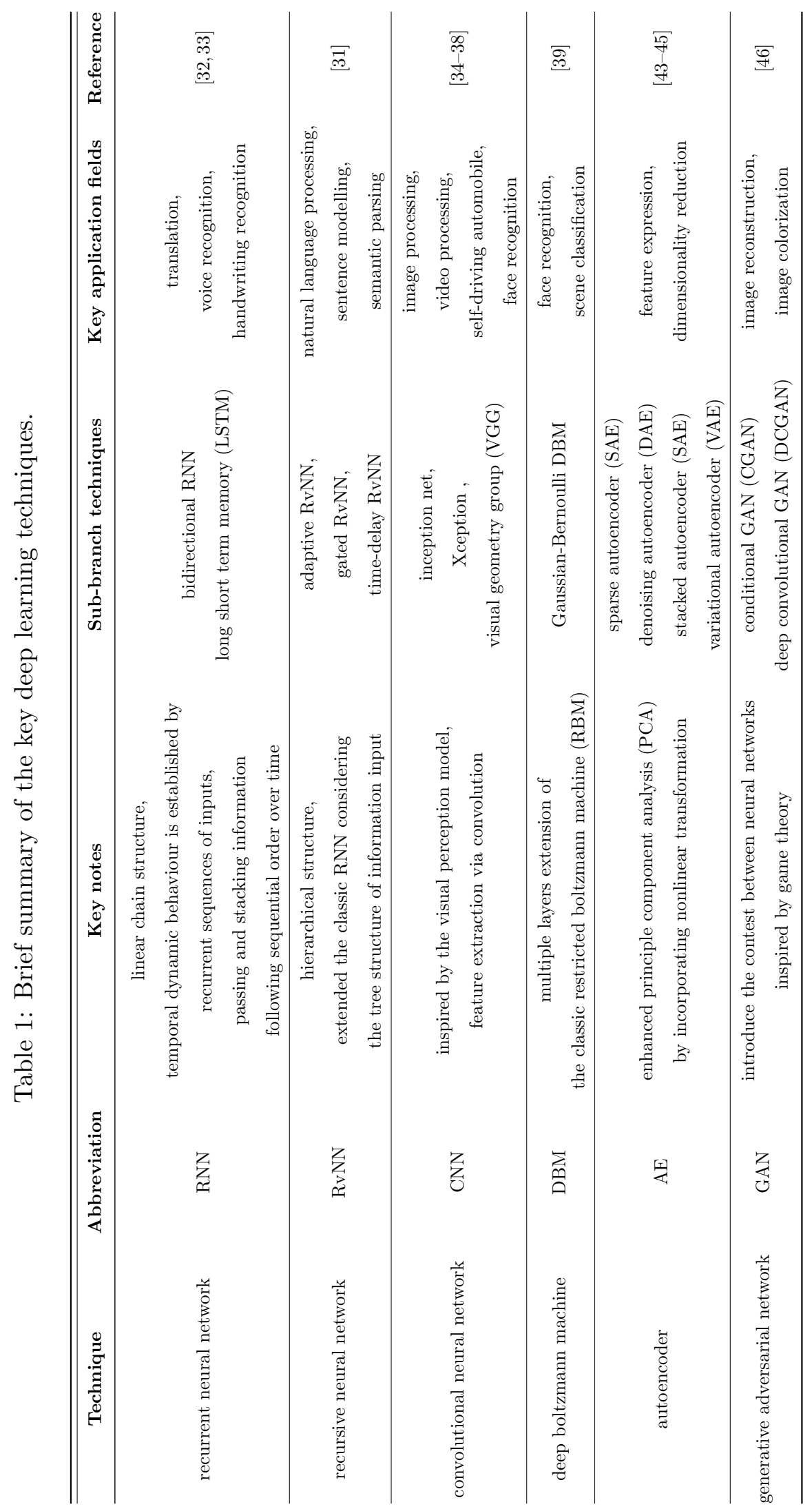


Based on the neural network algorithm, deep learning has been rapidly emerging and contains several popular techniques which are established over the recent years: recursive neural network $(\mathrm{RvNN})$ [31], recurrent neural network (RNN) [32], convolutional neural network (CNN) [37,38], deep boltzmann machine (DBM) [39], etc. A brief summary table of the popular key techniques and corresponding milestone literature are summarized in Table 1. Please note that more details of deep learning algorithms and frameworks can be found in [40-42] for readers who are interested in the technical aspect of deep learning, thus we will not reproduce here. One can also refer to deeplearning.net for abundant collections of resources.

\section{Implementations of Deep Learning in Banking}

The springing up of deep learning applications have been witnessed across sectors especially in medical diagnosis and biological data processing thank to the merits like pattern recognition and image processing deep learning has brought into these fields. After a comprehensive investigation of existing literature and other available resources online, considering the data-rich nature of banking sector and its generally welcoming approaches in terms of embracing datadriven technologies, this section aims to specifically investigate the existing implementations of deep learning in the high profile banking sector. By providing the most up to date summary of deep learning applications in banking, this paper contributes to connecting researchers in both domains and bringing insights on the future research directions and potential implementations.

According to the wide implementations of deep learning in a broader scale of sectors, it has been observed that the practical implementations of deep learning have many different forms, i.e. virtual service assistants, smart image processing, face recognition, medical diagnosis, personalized marketing, etc. The following subsections systematically summarise the applications of deep learning in practice into three domains of how deep learning is used for benefiting specifically the banking sector. Although practical implementation can be complex or presenting in the format of different combinations, we attempt to group the implementations into three domains including marketing, customer relationship management (CRM) and risk management. Specifically, each domain covers a variety of relevant activities so to assure the comprehensiveness of our review. 


\subsection{Marketing}

Deep learning has been strategically applied for marketing related activities across sectors [47], and the banking sector is no exception [48]. In general, deep learning can assist banks in targeting the "right" customers with "right" marketing campaign so to assure the effectiveness of its marketing activities under the intense competitions banks are facing nowadays.

Researchers have attempted to apply deep learning techniques in personalized marketing of retail banking, in which the most profitable group of customers are identified for corresponding marketing campaign, customer behaviours are also learned continuously so that banks can prioritize more precise offers and referrals. The telemarketing data of the Portugues banking institutions between 2008 and 2010 is used in [49] with deep CNN architecture for identifying customers who are likely to accept marketing proposal. The authors in [50] proposed a collection of modified artificial neural networks and applied on the bank direct marketing data set from [51] in verifying the performance in direct marketing. Yan (2018) [52] demonstrated the application of convolutional neural network via a Kaggle competition on Santander's customer data, in which the customer's behaviour are learned for predicting the usage of bank products. Ładyżyński et al. (2019) [53] used customer transactional data from a large retail bank in Poland and identified the group of more promising customers by deep belief networks and stacked restricted Boltzman machines via the $\mathrm{H} 2 \mathrm{O}$ platform, this then allows direct marketing and significantly improves the performance of marketing campaign.

\subsection{Customer Relationship Management (CRM)}

Just as any other customer oriented businesses, the banking sector also significantly value customer relationship management (CRM) given its intensely competitive business environment. As a data-rich sector which has access to abundant customer information as well as banking behaviour records, it is crucial ability for a bank to be able to understand their customer and offer timely customized service. By interacting with customers and being supportive with personalized services, the aim of successful CRM is to improve its productivity of customer interaction [54]. To the best of our knowledge, we here in this subsection list the observed applications of deep learning in assisting the CRM of banking sector. 
Customer profiling \& segmentation is the first important step of knowing your customer and is closely connected with direct marketing. The banks gather customer information systematically and transform all available data into a structured way so they are analyzable and ready for being exploited further by advanced techniques like deep learning. The banks are learning from the data obtained so to build up the comprehensive profile of their customer and potentially group their customers by different features and tasks. A relatively early research by Davies et al. (1996) [55] applied neural network analysis on customer segmentation in regard to one's attitudinal types against ATM services. Many research (i.e. $[56,57,62])$ followed and investigated customer segmentation via different models learning customer behaviour from content rich data collected by banks. Wang et al.(2018) [59] applied BP neural network algorithm on commercial banks' data set in China for establishing an improved customer hierarchy system. According to the customers data of the Saman Bank in Iran, Zhou et al.(2019) [60] compared the performance of neural network with other data mining techniques and achieved over $97 \%$ accuracy in customer behaviour segmentation.

Customer service and satisfaction is another main usage of deep learning in banking [61]. The advancements of deep learning enabled more intelligent chat-bot / service bot for offering restless customer services and improving customer satisfaction [62]. There have been many successful use cases of chat-bot across the world. For instance according to [63], Erica (the virtual assistant of Bank of America), COIN (contract intelligence platform of JPMorgan), Eno (chat-bot assistant of Captial One), Ally assistant (by Ally Bank), Clinc (by USAA), AmEx (by American Express), Amy (by HKBC), Haro and Dori (by Hang Seng Bank), Aida (by SEB), Emma (by OCBC Bank), Ceba (by Commonwealth Bank in Australia), POSB by (DBS bank), etc. These AI-driven chat-bots usually accept customer interactions via voice or text commands, as well as tapping options over the screen. They are intelligently engineered to give 24/7 efficient support for most of the day-to-day tasks of bank customers. Quah and Chuan (2019) [64] investigated the use of chat-bot in the banking industry of Singapore, which has been recognised as one of the top global FinTech hubs. The concern of trust as a challenge of applying chat-bot in banking is addressed in [65] with new measure proposed for evaluating chat-bot performance. 
Customer churn detection plays significant role in evaluating business success, customercentric sectors like banking also pay great attention on retaining customers. Thus, the improvements by embracing the most advanced techniques have been continuously observed on the identification of customer churn. Using the customer data set of a Chinese commercial bank, the authors in [66] addressed the advantage of deep learning algorithms on predicting customer churn. The transactional data of the private banks in Iran were adopted in [67] for verifying the capability of recurrent neural networks in preventing customer churn. A very recent research by Caigny et al.(2019). [68] systematically investigated the implementation of convolutional neural networks on customer churn prediction, and proved the improved performance of CNN in processing textual data.

It is noticed that considering the wide scale of CRM, its collaboration with deep learning techniques seems relatively limited, still many potentials are waiting to be discovered. It is possibly due to the fact that deep learning in comparison with general machine learning techniques has its merits mainly in image processing, natural language processing as well as audio/video processing. This determined that deep learning techniques in the format of chat-bot/service bot has the better premises of implementation in banking.

\subsection{Risk Management}

Another essential pillar of the banking sector is risk management. The general aim of risk management can be briefly summarized as protecting assets and preventing potential losses. For the banking sector which provides a variety of services covering almost every aspects of human life, its risk management meanwhile can be a difficult but crucial task. Therefore, it is undoubtedly that banking sector will actively embrace those advanced data analyses techniques for preventing risks to their business and assets. Parallel researches about general machine learning applications in banking risk management are conducted by Leo et al.(2019) [69] and Petropoulos et al.(2020) [70], which specifically focused on bank insolvencies prediction. An earlier research by Lin et al.(2011) [71] reviewed the machine learning applications for predicting financial crisis. In this subsection, we investigate specifically the implementations of deep learning techniques in some of the most important elements of risk management in banking, these include but not limited to: protecting the values of assets in investment, assessing risks of loan approval and preventing fraud. 
Investment and Portfolio Management It is of note that there have been relatively exhaustive research which focuses on the forecasting advancements of financial time series via deep learning techniques. More details can be found in a very recent comprehensive review paper of [72] in 2020, the authors systematically summarized over 200 applications between 2005 to 2019 , thus we will not reproduce the relevant literature. To assist the accurate evaluation of repricing risk, Karisma and Widyantoro (2016) [73] applied deep neural network algorithm on the monthly report data of public bank in Indonesia and achieved better identification of repricing gap than the standard backpropagation method. The usage of deep learning in option pricing was investigated by [74] and robust performance was confirmed comparing to the empirical approaches. Weigand (2019) [75] also focused on the asset pricing domain, and summarized the applications of general machine learning techniques. Chen et al.(2017) [76] applied the kcompetitive autoencoder deep learning techniques for text processing of financial reports made by US public banks and it outperformed the other machine learning techniques in terms of identifying failed bank and assisting financial decision making. Similarly, the authors in [77] used recurrent neural networks techniques and analysed over 6.6 million news articles for the prediction of bank distress. Using over 11 thousands US public companies data set, Mai et al.(2018) [78] compared the performances of convolutional neural networks and average embedding model in terms of predicting bankruptcy. Later, Qu et al.(2019) [79] presented a comprehensive review on the implementations of deep learning techniques on bankruptcy prediction. A recent study of Vo et al.(2019) [80] incorporated the environmental, social and governance metrics and the long short term memory (LSTM) deep learning technique for portfolio optimization with sustainable consideration of social impact.

Loan Approval It is of crucial importance for banks to comprehensively evaluate the risks before approving loans. Luo et al.(2016) [81] adopted the credit default swaps data set and applied deep belief networks and restricted boltzmann machines for achieving improved credit score modelling. A research project conducted by Sirignano et al. (2018) [82] used over 120 million mortgages data in the US between 1995 and 2014, and evaluated the performance of deep neural networks on modelling morgage risk. The authors in [83] compared the performances of tree based and artificial neural networks methods in predicting loan default probability of over 117 thousands enterprises. Kvamme et al.(2018) [84] investigated the real world Norwegian mortgage portfolio data set and applied convolutional neural networks for credit risk prediction. 
Fraud Detection The wide spread use of credit card has formed a significant portion of day to day banking services, which brings in both abundant profit as well as risks. The fraud detection is another important domain banks pay extreme attentions to in terms of preventing losses. In regard to the relevant implementations of deep learning techniques, the authors in [85] used deep artificial neural networks with 18 months of transactional data set for verifying the performance of fraud detection. Fu et al.(2016) [86] applied convolutional neural networks on real word transactional data set of a commercial bank in China and outperformed other established methods in identifying credit card fraud behaviours. With the same deep learning technique - convolutional neural network, similar task was conducted in [87] for online transaction fraud detection. A different deep learning technique - autoencoder was applied in [88] to distinguish credit card fraud from transactional data. In a similar research with autoencoder technique, data set from European banks was used in [89]. Pumsirirat and Yan (2018) [90] combined autoencoder and restricted boltzmann machine techniques for fraud detection experiments on German, Australian and European data set.

\section{Conclusion}

The springing up of technological advancements in data science has promoted significant changes across sectors in a global scale, this paper aims to provide further clarification of the emerging "deep learning" in the context of general confusions caused by its interchangeable use with other relevant concepts like AI and machine learning. The trends of deep learning indicates rapidly raising global interests, as such it is crucially important to provide clear introduction of deep learning and its key techniques. This contributes to overcome the general knowledge gap between technological experts and the general public, so to further promote its wide implementations. Here in this paper, we specifically investigated the banking sector due to its data-rich nature and historically welcoming environment in embracing advanced technologies. After the comprehensive review of most up to date deep learning applications in banking, to the best of our knowledge, it is noticed that deep learning techniques offers generally improved capacity in processing more complex data set, and more stable performance thank to the advancements of deep neural networks. However, the applications of deep learning in general have specialities in the domains of image processing and natural language processing. Therefore, its implementations in banking seem overall relatively limited (as being summarized in section 3), still many 
more potentials in the broader horizon of banking are waiting to be exploited. It is also noticed that there are relatively small amount of academic literature of deep learning in banking. Literature mainly investigate the general term machine learning and its applications, much less research that specifically study deep learning and its applications, not to mention focusing on a specific sector. Many of the most advanced techniques and its applications stay at the stage of conference presentation and proceeding, there is simply not enough reliable academic and public exposure to be widely acknowledged by the general public, which could be one of the reasons leading to knowledge gap. We discovered that the applications of deep learning in banking mainly targeted the customer relationship management and risk management domains, likely due to the availability of data in banking. There are also many potential deep learning implementations which we could not identify in existing academic literature in banking, for instance, face recognition, user authorization, cyber security, audio/video processing, etc. It is expected that this paper can connect the researchers and practitioners in both deep learning and banking fields and provide insights to future research.

\section{References}

[1] Hassani, H., Huang, X., Silva, E. S., \& Ghodsi, M. (2016). A review of data mining applications in crime. Statistical Analysis and Data Mining: The ASA Data Science Journal, 9(3), 139-154.

[2] Hassani, H., Huang, X., \& Ghodsi, M. (2018). Big data and causality. Annals of Data Science, $5(2), 133-156$.

[3] Hassani, H., Huang, X., \& Silva, E. (2018). Digitalisation and big data mining in banking. Big Data and Cognitive Computing, 2(3), 18.

[4] Hassani, H., Huang, X., \& Silva, E. (2019). Big Data and climate change. Big Data and Cognitive Computing, 3(1), 12

[5] Hassani, H., Huang, X., \& Silva, E. (2018). Banking with blockchain-ed big data. Journal of Management Analytics, 5(4), 256-275.

[6] Hormozi, A. M., \& Giles, S. (2004). Data mining: A competitive weapon for banking and retail industries. Information systems management, 21(2), 62-71. 
[7] Chitra, K., \& Subashini, B. (2013). Data mining techniques and its applications in banking sector. International Journal of Emerging Technology and Advanced Engineering, 3(8), 219226.

[8] Jayasree, V.,\& Balan, R. V. S. (2013). A review on data mining in banking sector. American Journal of Applied Sciences, 10(10), 1160.

[9] Chye, K. H., \& Gerry, C. K. L. (2002). Data mining and customer relationship marketing in the banking industry. Singapore Management Review, 24(2), 1-28.

[10] Aburrous, M., Hossain, M. A., Dahal, K., \& Thabtah, F. (2010). Intelligent phishing detection system for e-banking using fuzzy data mining. Expert systems with applications, 37(12), 7913-7921.

[11] Ince, H., \& Aktan, B. (2009). A comparison of data mining techniques for credit scoring in banking: A managerial perspective. Journal of Business Economics and Management, 10(3), 233-240.

[12] Sun, N., Morris, J. G., Xu, J., Zhu, X., \& Xie, M. (2014). iCARE: A framework for big data-based banking customer analytics. IBM Journal of Research and Development, 58(5/6), $4-1$.

[13] Srivastava, U., \& Gopalkrishnan, S. (2015). Impact of big data analytics on banking sector: Learning for Indian banks. Procedia Computer Science, 50, 643-652.

[14] Tsai, C. F., \& Chen, M. L. (2010). Credit rating by hybrid machine learning techniques. Applied soft computing, 10(2), 374-380.

[15] Khandani, A. E., Kim, A. J., \& Lo, A. W. (2010). Consumer credit-risk models via machinelearning algorithms. Journal of Banking \& Finance, 34(11), 2767-2787.

[16] Leo, M., Sharma, S., \& Maddulety, K. (2019). Machine learning in banking risk management: A literature review. Risks, 7(1), 29.

[17] Carr, M., Ravi, V., Reddy, G. S., \& Veranna, D. (2013). Machine learning techniques applied to profile mobile banking users in India. International Journal of Information Systems in the Service Sector (IJISSS), 5(1), 82-92.

[18] Smeureanu, I., Ruxanda, G., \& Badea, L. M. (2013). Customer segmentation in private banking sector using machine learning techniques. Journal of Business Economics and Management, 14(5), 923-939.

[19] Hassani, H., Huang, X., \& Silva, E. S. (2019). Fusing Big Data, Blockchain, and Cryptocurrency. In Fusing Big Data, Blockchain and Cryptocurrency. Palgrave Pivot. 
[20] Domingos, P. (2012). A few useful things to know about machine learning. Communications of the ACM, 55(10), 78-87.

[21] Grossfeld, B. (2020). Deep learning vs machine learning: a simple way to understand the difference. [Available online: https://https://www.zendesk.com/blog/machine-learning-anddeep-learning/][Accessed 20/03/2020].

[22] Alpaydin, E. (2020). Introduction to machine learning. MIT Press.

[23] Shalev-Shwartz, S., \& Ben-David, S. (2014). Understanding machine learning: From theory to algorithms. Cambridge University Press.

[24] Goodfellow, I., Bengio, Y., \& Courville, A. (2016). Deep learning. MIT Press.

[25] LeCun, Y., Bengio, Y., \& Hinton, G. (2015). Deep learning. Nature, 521(7553), 436-444.

[26] Guo, Y., Liu, Y., Oerlemans, A., Lao, S., Wu, S., \& Lew, M. S. (2016). Deep learning for visual understanding: A review. Neurocomputing, 187, 27-48.

[27] Zhu, X. X., Tuia, D., Mou, L., Xia, G. S., Zhang, L., Xu, F., \& Fraundorfer, F. (2017). Deep learning in remote sensing: A comprehensive review and list of resources. IEEE Geoscience and Remote Sensing Magazine, 5(4), 8-36.

[28] Miotto, R., Wang, F., Wang, S., Jiang, X., \& Dudley, J. T. (2018). Deep learning for healthcare: review, opportunities and challenges. Briefings in Bioinformatics, 19(6), 12361246.

[29] Fawaz, H. I., Forestier, G., Weber, J., Idoumghar, L., \& Muller, P. A. (2019). Deep learning for time series classification: a review. Data Mining and Knowledge Discovery, 33(4), 917-963.

[30] Fan, C., Xiao, F., \& Zhao, Y. (2017). A short-term building cooling load prediction method using deep learning algorithms. Applied Energy, 195, 222-233.

[31] Socher, R., Lin, C. C., Manning, C., \& Ng, A. Y. (2011). Parsing natural scenes and natural language with recursive neural networks. In Proceedings of the 28th international conference on machine learning (ICML-11) (pp. 129-136).

[32] Mikolov, T., Karafiát, M., Burget, L., Černockỳ, J., \& Khudanpur, S. (2010). Recurrent neural network based language model. In Eleventh annual conference of the international speech communication association.

[33] Hochreiter, S., \& Schmidhuber, J. (1997). Long short-term memory. Neural Computation, $9(8), 1735-1780$.

[34] Szegedy, C., Liu, W., Jia, Y., Sermanet, P., Reed, S., Anguelov, D., ... \& Rabinovich, A. (2015). Going deeper with convolutions. In Proceedings of the IEEE conference on computer vision and pattern recognition (pp. 1-9). 
[35] Simonyan, K., \& Zisserman, A. (2014). Very deep convolutional networks for large-scale image recognition. arXiv preprint arXiv:1409.1556.

[36] Chollet, F. (2017). Xception: Deep learning with depthwise separable convolutions. In Proceedings of the IEEE conference on computer vision and pattern recognition (pp. 12511258).

[37] LeCun, Y., Bottou, L., Bengio, Y., \& Haffner, P. (1998). Gradient-based learning applied to document recognition. Proceedings of the IEEE, 86(11), 2278-2324.

[38] Donahue, J., Jia, Y., Vinyals, O., Hoffman, J., Zhang, N., Tzeng, E., \& Darrell, T. (2014). Decaf: A deep convolutional activation feature for generic visual recognition. In International conference on machine learning (pp. 647-655).

[39] Salakhutdinov, R., \& Hinton, G. (2009). Deep boltzmann machines. In Artificial intelligence and statistics (pp. 448-455).

[40] Schmidhuber, J. (2015). Deep learning in neural networks: An overview. Neural Networks, 61, 85-117.

[41] Pouyanfar, S., Sadiq, S., Yan, Y., Tian, H., Tao, Y., Reyes, M. P., Shyu, M.L., Chen, S.C., \& Iyengar, S. S. (2018). A survey on deep learning: Algorithms, techniques, and applications. ACM Computing Surveys (CSUR), 51(5), 1-36.

[42] Liu, W., Wang, Z., Liu, X., Zeng, N., Liu, Y., \& Alsaadi, F. E. (2017). A survey of deep neural network architectures and their applications. Neurocomputing, 234, 11-26.

[43] Bengio, Y., Lamblin, P., Popovici, D., \& Larochelle, H. (2007). Greedy layer-wise training of deep networks. In Advances in neural information processing systems (pp. 153-160).

[44] Vincent, P., Larochelle, H., Bengio, Y., \& Manzagol, P. A. (2008). Extracting and composing robust features with denoising autoencoders. In Proceedings of the 25th international conference on Machine learning (pp. 1096-1103).

[45] Kingma, D. P., \& Welling, M. (2013). Auto-encoding variational bayes. arXiv preprint arXiv:1312.6114.

[46] Goodfellow, I., Pouget-Abadie, J., Mirza, M., Xu, B., Warde-Farley, D., Ozair, S., ... \& Bengio, Y. (2014). Generative adversarial nets. In Advances in neural information processing systems (pp. 2672-2680).

[47] Bose, I., \& Chen, X. (2009). Quantitative models for direct marketing: A review from systems perspective. European Journal of Operational Research, 195(1), 1-16. 
[48] Sing'oei, L., \& Wang, J. (2013). Data mining framework for direct marketing: A case study of bank marketing. International Journal of Computer Science Issues (IJCSI), 10(2 Part 2), 198.

[49] Kim, K. H., Lee, C. S., Jo, S. M., \& Cho, S. B. (2015). Predicting the success of bank telemarketing using deep convolutional neural network. In 2015 7th International Conference of Soft Computing and Pattern Recognition (SoCPaR) (pp. 314-317). IEEE.

[50] Zakaryazad, A., \& Duman, E. (2016). A profit-driven Artificial Neural Network (ANN) with applications to fraud detection and direct marketing. Neurocomputing, 175, 121-131.

[51] Moro, S., Cortez, P., \& Rita, P. (2014). A data-driven approach to predict the success of bank telemarketing. Decision Support Systems, 62, 22-31.

[52] Yan, C. (2018). Convolutional Neural Network on a structured bank customer data. Towards data science. [Available online: https://towardsdatascience.com/convolutional-neuralnetwork-on-a-structured-bank-customer-data-358e6b8aa759][accessed on 25/03/2020].

[53] Ładyżyński, P., Żbikowski, K., \& Gawrysiak, P. (2019). Direct marketing campaigns in retail banking with the use of deep learning and random forests. Expert Systems with Applications, $134,28-35$.

[54] Ogwueleka, F. N., Misra, S., Colomo-Palacios, R., \& Fernandez, L. (2015). Neural network and classification approach in identifying customer behavior in the banking sector: A case study of an international bank. Human factors and ergonomics in manufacturing \& service industries, 25(1), 28-42.

[55] Davies, F., Moutinho, L., \& Curry, B. (1996). ATM user attitudes: a neural network analysis. Marketing Intelligence \& Planning, 14, 26-32.

[56] Hsieh, N. C. (2004). An integrated data mining and behavioral scoring model for analyzing bank customers. Expert systems with applications, 27(4), 623-633.

[57] Laukkanen, T., \& Pasanen, M. (2008). Mobile banking innovators and early adopters: How they differ from other online users?. Journal of Financial Services Marketing, 13(2), 86-94.

[58] Krishna, G. J., Ravi, V., Reddy, B. V., Zaheeruddin, M., Jaiswal, H., Teja, P. S. R., \& Gavval, R. (2019, October). Sentiment Classification of Indian Banks' Customer Complaints. In TENCON 2019-2019 IEEE Region 10 Conference (TENCON) (pp. 429-434). IEEE.

[59] Wang, G., Bai, Y., \& Sun, Y. (2018). Application of BP neural network algorithm in bank customer hierarchy system. In 2017 3rd International Forum on Energy, Environment Science and Materials (IFEESM 2017). Atlantis Press. 
[60] Zhou, X., Bargshady, G., Abdar, M., Tao, X., Gururajan, R., \& Chan, K. C. (2019). A case study of predicting banking customers behaviour by using data mining. In 2019 6th International Conference on Behavioral, Economic and Socio-Cultural Computing (BESC) (pp. 1-6). IEEE.

[61] Vieira, A., \& Sehgal, A. (2018). How banks can better serve their customers through artificial techniques. In Digital Marketplaces Unleashed (pp. 311-326). Springer, Berlin, Heidelberg.

[62] Krishnan, K. (2020). chapter 7 - Banking industry applications and usage. Building Big Data Applications, pp. 127-144. Academic Press.

[63] Marous, J. (2018). Meet 11 of the most interesting chatbots in banking. The Financial Brand. [Available online: https://thefinancialbrand.com/71251/chatbots-banking-trends-aicx/][accessed on 26/03/2020].

[64] Quah, J. T., \& Chua, Y. W. (2019). Chatbot Assisted Marketing in Financial Service Industry. In International Conference on Services Computing (pp. 107-114). Springer.

[65] Przegalinska, A., Ciechanowski, L., Stroz, A., Gloor, P., \& Mazurek, G. (2019). In bot we trust: A new methodology of chatbot performance measures. Business Horizons, 62(6), 785-797.

[66] Spanoudes, P., \& Nguyen, T. (2017). Deep learning in customer churn prediction: unsupervised feature learning on abstract company independent feature vectors. arXiv preprint arXiv:1703.03869.

[67] Mirashk, H., Albadvi, A., Kargari, M., Javide, M., Eshghi, A., \& Shahidi, G. (2019). Using RNN to Predict Customer Behavior in High Volume Transactional Data. In International Congress on High-Performance Computing and Big Data Analysis (pp. 394-405). Springer.

[68] De Caigny, A., Coussement, K., De Bock, K. W., \& Lessmann, S. (2019). Incorporating textual information in customer churn prediction models based on a convolutional neural network. International Journal of Forecasting. In Press.

[69] Leo, M., Sharma, S., \& Maddulety, K. (2019). Machine learning in banking risk management: A literature review. Risks, 7(1), 29.

[70] Petropoulos, A., Siakoulis, V., Stavroulakis, E., \& Vlachogiannakis, N. E. (2020). Predicting bank insolvencies using machine learning techniques. International Journal of Forecasting. In Press. [Available online: https://doi.org/10.1016/j.ijforecast.2019.11.005]. 
[71] Lin, W. Y., Hu, Y. H., \& Tsai, C. F. (2011). Machine learning in financial crisis prediction: a survey. IEEE Transactions on Systems, Man, and Cybernetics, Part C (Applications and Reviews), 42(4), 421-436.

[72] Sezer, O. B., Gudelek, M. U., \& Ozbayoglu, A. M. (2020). Financial time series forecasting with deep learning: A systematic literature review: 2005-2019. Applied Soft Computing, 106181.

[73] Karisma, H., \& Widyantoro, D. H. (2016). Comparison study of neural network and deep neural network on repricing GAP prediction in Indonesian conventional public bank. In 2016 6th International Conference on System Engineering and Technology (ICSET) (pp. 116-122). IEEE.

[74] Culkin, R., \& Das, S. R. (2017). Machine learning in finance: the case of deep learning for option pricing. Journal of Investment Management, 15(4), 92-100.

[75] Weigand, A. (2019). Machine learning in empirical asset pricing. Financial Markets and Portfolio Management, 33(1), 93-104.

[76] Chen, Y., Rabbani, R. M., Gupta, A., \& Zaki, M. J. (2017). Comparative text analytics via topic modeling in banking. In 2017 IEEE Symposium Series on Computational Intelligence (SSCI) (pp. 1-8). IEEE.

[77] Rönnqvist, S., \& Sarlin, P. (2017). Bank distress in the news: Describing events through deep learning. Neurocomputing, 264, 57-70.

[78] Mai, F., Tian, S., Lee, C., \& Ma, L. (2019). Deep learning models for bankruptcy prediction using textual disclosures. European Journal of Operational Research, 274(2), 743-758.

[79] Qu, Y., Quan, P., Lei, M., \& Shi, Y. (2019). Review of bankruptcy prediction using machine learning and deep learning techniques. Procedia Computer Science, 162, 895-899.

[80] Vo, N. N., He, X., Liu, S., \& Xu, G. (2019). Deep learning for decision making and the optimization of socially responsible investments and portfolio. Decision Support Systems, $124,113097$.

[81] Luo, C., Wu, D., \& Wu, D. (2017). A deep learning approach for credit scoring using credit default swaps. Engineering Applications of Artificial Intelligence, 65, 465-470.

[82] Sirignano, J., Sadhwani, A., \& Giesecke, K. (2018). Deep Learning for Mortgage Risk. [Available at: http://dx.doi.org/10.2139/ssrn.2799443].

[83] Addo, P. M., Guegan, D., \& Hassani, B. (2018). Credit risk analysis using machine and deep learning models. Risks, 6(2), 38 . 
[84] Kvamme, H., Sellereite, N., Aas, K., \& Sjursen, S. (2018). Predicting mortgage default using convolutional neural networks. Expert Systems with Applications, 102, 207-217.

[85] Gomez, J. A., Arevalo, J., Paredes, R., \& Nin, J. (2018). End-to-end neural network architecture for fraud scoring in card payments. Pattern Recognition Letters, 105, 175-181.

[86] Fu, K., Cheng, D., Tu, Y., \& Zhang, L. (2016). Credit card fraud detection using convolutional neural networks. In International Conference on Neural Information Processing (pp. 483-490). Springer.

[87] Zhang, Z., Zhou, X., Zhang, X., Wang, L., \& Wang, P. (2018). A model based on convolutional neural network for online transaction fraud detection. Security and Communication Networks, 2018.

[88] Kazemi, Z., \& Zarrabi, H. (2017). Using deep networks for fraud detection in the credit card transactions. In 2017 IEEE 4th International Conference on Knowledge-Based Engineering and Innovation (KBEI) (pp. 0630-0633). IEEE.

[89] Zamini, M., \& Montazer, G. (2018). Credit card fraud detection using autoencoder based clustering. In 2018 9th International Symposium on Telecommunications (IST) (pp. 486-491). IEEE.

[90] Pumsirirat, A., \& Yan, L. (2018). Credit card fraud detection using deep learning based on auto-encoder and restricted boltzmann machine. International Journal of advanced computer science and applications, 9(1), 18-25. 